\title{
Israelfeindschaft und Antisemitismus bei Hamas, Al-Qaida und Islamischem Staat
}

\author{
Michel Wyss
}

Während seiner Rede auf der jährlichen UN-Generalversammlung Ende September 2014 sorgte der israelische Ministerpräsident Benjamin Netanjahu für Aufregung. Nur wenigen Wochen nach Ende des jüngsten Waffengangs zwischen Israel und der Hamas in Gaza und einige Monate nachdem der Islamische Staat Mossul und weite Teil des Iraks unter seine Kontrolle gebracht hatte, erklärte Netanjahu vor versammelter Weltöffentlichkeit:

„Last week, many of the countries represented here rightly applauded President Obama for leading the effort to confront ISIS, and yet weeks before, some of these same countries, the same countries that now support confronting ISIS, opposed Israel for confronting Hamas. They evidently don't understand that ISIS and Hamas are branches of the same poisonous tree. [...] So when it comes to their ultimate goals, Hamas is ISIS and ISIS is Hamas. And what they share in common, all militant Islamists share in common. Boko Haram in Nigeria, Al-Shabab in Somalia, Hezbollah in Lebanon, AlNusra in Syria, the Mahdi army in Iraq, and the Al-Qaida branches in Yemen, Libya, the Philippines, India and elsewhere. “1

Bereits vor dem UN-Gipfel hatten Netanjahu und das israelische Außenministerium die Kernbotschaft „Hamas ist ISIS und ISIS ist Hamas“ sowohl in den sozialen Medien als auch bei öffentlichen Auftritten lanciert und beispielsweise die Massenermordung schiitisch-irakischer Soldaten im Camp Speicher bei Tikrit durch den Islamischen Staat mit der Tötung von vermeintlichen Kollaborateuren in Gaza durch die Hamas verglichen. ${ }^{2}$ Während seine Regierungskoalition applaudierte, wurde Netanjahus Gleichsetzung von verschiedenen Seiten vehement kritisiert. ${ }^{3}$ Einer der gängigen Vorwürfe lautete, Netanjahu wolle politisch punkten und würde die Gräueltaten des Islamischen Staates auf Kosten der Hamas instrumentalisieren. Die Hamas wiederum

1 Transcript of Benjamin Netanyahu's Address to the 2014 UN General Assembly, in: Haaretz, 29.9.2014, [http://www.haaretz.com/israel-news/1.618308], eingesehen 13.8.2017.

2 Yoni Kempinski/Gil Ronen, Netanyahu: Hamas is ISIS, ISIS is Hamas, in: Arutz. Sheva, 21.8.2014, [http://www.israelnationalnews.com/News/News.aspx/184288], eingesehen 13.8.2017; Israel's Foreign Affairs Min., Hamas is ISIS - ISIS is Hamas, in: YouTube, 25.8.2014, [https://youtu.be/Eza9OtoH60A], eingesehen 13.8.2017.

3 Washington rejects Netanyahu's comparison of Iran and Hamas to ISIS, in: Middle East Monitor, 30.9.2014, [https://www.middleeastmonitor.com/20140930-washington-rejects-netanyahus-comparison-of-iran-and-hamas-toisis/], eingesehen 13.8.2014; Yossi Mekelberg, No Mr. Netanyahu, Hamas is not ISIS, in: Al-Arabiya, 17.9.2014, [https://english.alarabiya.net/en/views/news/middle-east/2014/09/17/No-Mr-Netanyahu-Hamas-is-not-

ISIS.html], eingesehen 13.8.2017; Max Fisher, Hamas isn't ISIS. Here's why Netanyahu says it anyway, in: Vox, 25.8.2014, [https://www.vox.com/2014/8/25/6064467/no-netanyahu-hamas-is-not-isis-isis-is-not-hamas], eingesehen 13.8.2017. 
protestierte ebenfalls energisch und Politbüro-Chef Khaled Meshaal ließ in einem Interview mit Yahoo News verlauten, dass die de facto Machthaberin in Gaza keine gewalttätige religiöse Organisation sei. ${ }^{4}$ Bis auf einige wenige Ausnahmen, machten sich Netanjahus Kritiker und Kritikerinnen allerdings nicht die Mühe, sich inhaltlich mit seiner These auseinanderzusetzen und zu erörtern, ob und inwiefern Hamas, der Islamische Staat und weitere dschihadistische Organisation miteinander gleichzusetzen seien. ${ }^{5}$ Ziel dieses Beitrags ist es deshalb, mittels einer vergleichenden Fallstudie sowohl Gemeinsamkeiten als auch Gegensätze zwischen Hamas und Islamischem Staat herauszuarbeiten. Neben Hamas und Islamischem Staat wird die Fallstudie mit Al-Qaida auch die Mutterorganisation der Globalen Dschihad-Bewegung einbeziehen, da sie mit den beiden erstgenannten Organisationen langjährige, komplexe Beziehungen aufweist, welche permanent zwischen Kooperation und Konkurrenz pendeln. Aus Gründen der Vergleichbarkeit fokussiert die Studie damit ausschließlich auf sunnitischdschihadistische Gruppierungen und berücksichtigt keine schiitischen Organisationen wie Hezbollah oder Mahdi-Armee, welche zweifelsohne einer separaten Betrachtung wert wären. Im Einklang mit dem thematischen Schwerpunkt dieses Sammelbands legt die Fallstudie ein besonderes Augenmerk auf die Bedeutung von Antisemitismus und Antizionismus für Hamas, Al-Qaida und Islamischen Staat. Dieser Fokus bietet sich aus mehreren Gründen an. Zum einen sind Juden- und Israelfeindschaft zweifelsohne ein zentrales Element islamistischer und dschihadistischer Ideologie. ${ }^{6}$ Zum anderen eignet sich diese Schwerpunktsetzung besonders gut, um einige der grundsätzlichen Unterschiede zwischen Hamas, Al-Qaida und Islamischem Staat anhand zweier spezifischer Merkmale zu illustrieren. Wie sich im Folgenden zeigen wird, ist die Forschungsfrage, ob und inwiefern Hamas, Al-Qaida und Islamischer Staat miteinander gleichzusetzen sind, weniger eindeutig zu beantworten als sich dies Gegner wie Befürworter von Netanjahus „ISIS ist Hamas, Hamas ist ISIS“-These wünschen würden. Zum Zweck dieses Beitrags wird Antisemitismus als „Feindschaft gegen Juden als Juden“ definiert. $^{7}$ Antizionismus wiederum ist ,the denial of the right of Jews to be part of a worldwide community or to constitute an independent political unit in Israel" ${ }^{\text {" }}$, beziehungsweise, in den Worten von Shlomo Avineri, ,a fundamental refusal to accept the legitimacy of Israel“"?

\footnotetext{
${ }^{4}$ Michael Isikoff, Hamas leader: Don't compare us to ISIL, in: Yaboo News, 22.8.2014, [https://www.yahoo.com/news/hamas-leader--don-t-compare-us-to-isil-193125056.html], eingesehen 13.8.2017. ${ }_{5}$ Eine der wenigen fundierten Auseinandersetzungen fand sich in der Times of Israel: Lazar Berman, Is Hamas Really ISIS, in: The Times of Israel, 27.8.2014, [http://www.timesofisrael.com/is-hamas-really-isis/], eingesehen 14.8.2017.

6 Bassam Tibi, Religion, Prejudice and Annihilation. The Case of Traditional Islamic Judeophobia and Its Transformation into the Modern Islamist Antisemitism, in: Anthony McElligott/Jeffrey Herf (Hrsg.), Antisemitism Before and Since the Holocaust. Altered Contexts and Recent Perspectives, Cham 2017, S. 115-145.

${ }^{7}$ Armin Pfahl-Traughber, Antisemitismus als Feindschaft gegen Juden als Juden, in: Der Bürger im Staat 63 (2013), Heft 4, S. 252-261.

8 Yehuda Bauer, Antisemitism and Anti-Zionism - New and Old, in: Robert S. Wistrich (Hrsg.), Anti-Zionism and Antisemitism in the Contemporary World, London 1990, S. 195-207, hier S. 198-199.

${ }^{9}$ Shlomo Avineri, Western Anti-Zionism. The Middle Ground, in: Robert S. Wistrich (Hrsg.), Anti-Zionism and
} 
Mittlerweile gibt es eine Reihe an ausführlichen Studien zu Hamas, Al-Qaida und insbesondere dem Islamischen Staat, sowohl auf Deutsch als auch Englisch. ${ }^{10}$ Vergleichende Fallstudien zu den drei Organisationen sind aber selten zu finden, insbesondere nicht auf Deutsch. ${ }^{11}$ Auch gibt es kaum Arbeiten zum Thema, die explizit auf die Bedeutung von Antisemitismus und Antizionismus für die jeweilige Gruppierung fokussieren, insbesondere nicht bei Al-Qaida und dem Islamischen Staat. ${ }^{12}$

Dieser Beitrag ist in fünf Abschnitte unterteilt. Der erste Abschnitt zeichnet in aller Kürze die Entwicklung des politischen Islam seit Ende des 19. Jahrhunderts bis zur Entstehung der Globalen Dschihad-Bewegung in den 1980er-Jahren nach. Die folgenden Abschnitte sind den eigentlichen Fallstudien von Antizionismus und Antisemitismus bei Hamas, Al-Qaida und dem Islamischen Staat gewidmet (in dieser Reihenfolge). Die Fallstudien beginnen mit einem kurzen historischen Überblick der jeweiligen Organisation und fokussieren danach auf die Bedeutung von Antizionismus und Antisemitismus für die entsprechende Gruppierung, sowohl für deren Ideologie als auch bezüglich konkreten israelfeindlichen oder antisemitisch motivierten Aktivitäten (die von Rhetorik und Propaganda bis zu Terroranschlägen und Guerillakriegsführung reichen). Der Beitrag schließt mit einem Vergleich und abschließenden Überlegungen.

Antisemitism in the Contemporary World, London 1990, S. 171-177, hier S. 171.

${ }_{10}$ Joseph Croituru, Hamas. Der Islamische Kampf um Palästina, München 2007; Matthew Levitt, Hamas. Politics, Charity, and Terrorism in the Service of Jihad, New Haven 2006; Peter Wichmann, Al-Qaida und der Globale Djihad. Eine vergleichende Betrachtung des transnationalen Terrorismus, Wiesbaden 2014; Bruce Hoffman/Fernando Reinares (Hrsg.), The Evolution of the Global Terrorist Threat. From 9/11 to Osama Bin Laden's Death, New York 2014; Barak Mendelsohn, The al-Qaeda Franchise. The Expansion of Al-Qaeda and Its Consequences, New York 2016; Guido Steinberg, Kalifat des Schreckens. IS und die Bedrohung durch den islamistischen Terror, München 2015; Christoph Reuter, Die Schwarze Macht. Der „Islamische Staat“ und die Strategen des Terrors, München 2015; Behnam T. Said, Islamischer Staat. IS-Miliz, al-Qaida und die deutschen Brigaden, München 2015; Wilfried Buchta, Terror vor Europas Toren. Der Islamische Staat, Iraks Zerfall und Amerikas Ohnmacht, Frankfurt a. M.-New York 2015; Peter Neumann, Die neuen Dschihadisten. ISIS, Europa und die nächste Welle des Terrorismus, Berlin 2015; Jessica Stern/J.M. Berger, ISIS. The State of Terror, New York 2015; Michael Weiss/Hassan Hassan, ISIS. Inside the Army of Terror, London-New York 2015; William McCants, The ISIS Apocalypse. The History, Strategy, and Doomsday Vision of the Islamic State, New York 2015; Patrick Cockburn, The Rise of Islamic State. ISIS and the New Sunni Revolution, Brooklyn 2015; Joby Warrick, Black Flags: The Rise of ISIS, New York 2015; Daniel Byman, Al Qaeda, the Islamic State, and the Global Jihad Movement. What Everybody Needs to Know, New York 2015; Fawaz A. Gerges, ISIS. A History, Princeton 2016.

${ }^{11}$ Eine der wenigen Ausnahmen ist Reuven Paz' Vergleich von Hamas und Al-Qaida, siehe Reuven Paz, Jihadis and Hamas, in: Assaf Moghadam/Brian Fishman (Hrsg.), Self-Inflicted Wounds. Debates and Divisions within Al-Qa'ida and its Periphery, West Point 2010, S. 183-201.

$12 \mathrm{Zu}$ Antisemitismus bei der Hamas, siehe Meir Litvak, The Antisemitism of Hamas, in: Palestine-Israel Journal 12 (2005), Heft 2/3, S. 41-46; Günther Jikeli, A Framework for Assessing Antisemitism. Three Case Studies (Dieudonné, Erdoğan, and Hamas), in: Alvin Rosenfeld (Hrsg.), Deciphering the New Antisemitism, Bloomington 2015, S. 43-76. 


\section{Von der Muslimbruderschaft zum Globalen Dschihad: Die Evolution des politischen Islam}

Gegen Ende des 19. Jahrhunderts stand die islamische Welt - insbesondere in ihrem Kerngebiet, dem Nahen Osten - vor einem Trümmerhaufen. Wenige Jahrhunderte zuvor Europa noch in fast allen Bereichen überlegen, hatte sie nun militärisch, technologisch wie kulturell das Nachsehen. ${ }^{13}$ Muslimische Intellektuelle wie Jamal AlDin Al-Afghani, Muhammad Abdu, sowie zu Beginn Abdus Schüler Rashid Rida waren überzeugt davon, dass eine Modernisierung des Islam nicht bloß von wörtlicher Interpretation und blinder Imitation religiöser Praktiken Abstand nehmen, sondern auch vom Westen lernen musste, insbesondere im Bereich der Wissenschaften. ${ }^{14} \mathrm{Nach}$ Abdus Tod wandte sich Rida allerdings einer puritanischen Version des Islam zu, d. h. einer strikten wörtlichen Interpretation von Koran und Sunna im Stile Ibn Taymiyyas, bis heute einem der wichtigsten Stichwortgeber für dschihadistische Ideologie und Ideologen. ${ }^{15}$ Rida war auch einer der ersten muslimischen Intellektuellen, die sich mit dem Zionismus beschäftigten. ${ }^{16}$ Seine anfängliche Bewunderung für die Juden und den Zionismus, als Vorbild für die islamische Gemeinschaft, schlug allerdings wenige Jahre später in offene Feindschaft um. ${ }^{17}$ So war Rida zunehmend davon überzeugt, dass es sich beim Kampf zwischen Zionisten und Arabern um einen religiösen Krieg handelte, den die Muslime gegen die „verräterischen Juden“ letzten Endes gewinnen würden. ${ }^{18}$ Ridas Überlegungen zum Zionismus beeinflussten den gerade in seiner Entstehung begriffenen politischen Islam nachhaltig und wurden etwa von Hassan Al-Banna übernommen. ${ }^{19} \mathrm{Al}-$ Banna, der 1928 in Ismailiyya die ägyptische Muslimbruderschaft gründete, kann mit Fug und Recht als einer der Urväter des politischen Islam bezeichnet werden. Al-Bannas Programm war simpel und effektiv: „Der Islam ist die Lösung.“ Die islamische Welt könne ihre einstige Vormachtstellung erst dann zurückerhalten, wenn sich die Muslime auf die Lehren des Islam besinnen würden. ${ }^{20}$ Al-Banna verstand es, eine einfache Sprache mit sozialer Wohlfahrt und religiöser Indoktrinierung $\mathrm{zu}$ verbinden, was die Muslimbruderschaft insbesondere für die ärmeren und weniger gebildeten Schichten Ägyptens attraktiv machte - eine Art „Islamisierung von unten“. Doch obwohl religiöse Bekehrung (arab. Da'wa, der „Ruf zum Islam“) seit jeher zum wichtigsten Instrument der Muslimbruderschaft zählte, beschränkte sie sich auch zu Zeiten Al-Bannas nicht darauf. Al-Banna selbst betonte die Bedeutung von gewalttätigem Dschihad für die

${ }^{13}$ Bernard Lewis, What Went Wrong. The Clash Between Islam and Modernity in the Middle East, New York 2003.

${ }^{14}$ Bernard Haykel, On the Nature of Salafi Thought and Action, in: Roel Mejer (Hrsg.), Global Salafism. Islam's New

Religious Movement, Oxford 2014, S. 33-57, hier S. 45-46.

15 Ebd., 46.

${ }^{16}$ Uriya Shavit, Zionism as told by Rashid Rida, in: Journal of Israeli History 34 (2015), Heft 1, S. $23-44$.

${ }^{17}$ Shavit, Zionism as told by Rashid Rida, S. 29-32.

18 Ebd., S. 38.

${ }^{19}$ Ebd., S. 39-40.

${ }^{20}$ Richard Paul Mitchell, The Society of the Muslim Brothers, New York 1993, S. 232. 
Verteidigung des Islam und erlaubte zu Beginn der 1940er-Jahre den Aufbau einer geheimen paramilitärischen Truppe, der nizam al-khass (Spezialabteilung) bzw. al-jihaz al-sirri (Geheimorganisation). ${ }^{21}$ Mitglieder der Geheimorganisation waren mutmaßlich verantwortlich für zahlreiche Gewaltakte wie etwa Bombenattentate, die Ermordung von politischen Rivalen sowie Angriffe auf britische Truppen. ${ }^{22}$ Diese Umtriebe führten 1948 zum ersten von mehreren Verboten der Muslimbruderschaft. Doch trotz Al-Bannas Dschihad-Rhetorik und seinem Bestreben, in Ägypten eine islamische Ordnung basierend auf der Scharia zu etablieren, bzw. „im islamischen Vaterland frei von ausländischem Einfluss einen islamischen Staat $\mathrm{zu}$ schaffen“, lehnte die Muslimbruderschaft Konzepte wie den modernen Nationalstaat oder Demokratie nicht $\mathrm{ab}$, sondern argumentierte, dass diese mit der islamischen Lehre vereinbar seien (allerdings interpretierte Al-Banna Konzepte wie Demokratie und Patriotismus gemäß seinem Islamverständnis um). ${ }^{23}$ Al-Banna nahm selbst an den ägyptischen Präsidentschaftswahlen teil (die er verlor) und die Ableger der Muslimbruderschaft in verschiedenen islamischen Ländern formten Parteien und nahmen an Wahlen teil (allerdings gab es durchaus Unterschiede; die Muslimbruderschaft in Syrien war etwa wesentlich radikaler als die Ennahda in Tunesien). Die Muslimbruderschaft und ihre Ableger erkannten damit nicht nur die Legitimität von Nationalstaaten an, sie sprachen sich auch meist für einen „Dialog mit dem Westen“ aus (ohne dies genauer zu konkretisieren). Explizit davon ausgenommen war Israel, gegen das auch vermeintlich „moderate“ Islamisten wie Rachid Ghannouchi, der Vorsitzende der Ennahda-Partei in Tunesien, wiederholt zum Dschihad aufriefen. ${ }^{24}$ Die - zumindest vorgebliche Akzeptanz der auf nationalstaatlicher Souveränität beruhenden Weltordnung und Dialogbereitschaft mit dem Westen sind die entscheidenden Unterschiede zwischen der Muslimbruderschaft und der globalen Dschihad-Bewegung. ${ }^{25}$ Letztere lehnt beide Ideen, wie überhaupt jegliche Art von Politik, die nicht allein auf der Scharia basiert, konsequent ab. Einzig und allein der bewaffnete Kampf sowie religiöse Bekehrung zur Etablierung eines globalen Kalifates sind für sie akzeptabel. ${ }^{26}$ Ironischerweise war es aber ausgerechnet ein Muslimbruder, der die Entstehung der globalen Dschihad-Bewegung maßgeblich mitbeeinflusste. Sayyid Qutb, Literaturkritiker und Lehrer, hatte zwei Jahre in den USA verbracht, um das dortige Erziehungssystem zu studieren, zeigte sich aber von den amerikanischen Umgangsformen angewidert und schockiert. ${ }^{27} \mathrm{Nach}$ seiner

${ }^{21}$ Ebd., S. 30.

22 Ebd., S. 58-65.

23 Nelly Lahoud, The Evolution of Modern Jihadism, in: The Oxford Research Encyclopdia of Religion, August 2016, [http://doi.org/10.1093/acrefore/9780199340378.013.68], eingesehen 13.8.2017.

24 The Global Muslim Brotherhood Daily Watch, Rachid Ghannouchi, o. D.,

[https://www.globalmbwatch.com/rachid-ghannouchi/], eingesehen 21.9.2017.

${ }^{25}$ Lahoud, The Evolution of Modern Jihadism.

26 Ebd.

${ }^{27}$ Sayyid Qutb, The America I Have Seen. In the Scale of Human Values, 1951,

[https://archive.org/stream/SayyidQutb/The\%20America\%20I\%20have\%20seen_djvu.txt], eingesehen 4.11.2017; 
Rückkehr nach Ägypten trat er in den frühen 1950er-Jahren in die Muslimbruderschaft ein und wurde zu einem der einflussreichsten Theoretiker des politischen Islam. Qutb argumentierte, dass sich die islamische Gemeinschaft in einer erneuerten Phase der „jahiliyya“ (arab. Ignoranz, der Begriff bezeichnete auch die vor-islamische Periode) befinde, da sie in Ländern lebe, die nicht der Scharia folgten. Gemäß Qutb hätten einzig die Gesetze Allahs Legitimität, alle anderen widersprächen hingegen dessen Souveränität. ${ }^{28}$ Qutb bestand darauf, dass der Koran ewige Gültigkeit habe und nicht in einem bestimmten historischen Kontext verstanden werden müsse. Aus diesem Grunde würden auch die Beschreibungen von Juden als feige und verräterisch heute noch genauso zutreffen wie zu den Zeiten Muhammads. ${ }^{29}$ Schließlich argumentierte Qutb, dass Dschihad der allumfassende (physische wie mentale) Kampf gegen alles sei, was der Anbetung Allahs widerspreche. Dieser Kampf ist gemäß Qutb nicht nur gegen Ungläubige zu führen, sondern auch gegen jene Muslime, die vom „rechten Glauben“ abgefallen sind. Qutb ging noch einen Schritt weiter und erklärte die Tötung von muslimischen Staatsoberhäuptern für rechtens, wenn diese nicht gemäß der Scharia regierten. Die Exkommunikation (takfir) von Muslimen als Rechtfertigung für ihre Tötung wurde von späteren Theoretikern der Dschihad-Bewegung von Staatsoberhäuptern auf sämtliche Muslime ausgeweitet, bleibt aber umstritten. ${ }^{30}$

Aufgrund seiner radikalen Ansichten wurde Qutb 1954 (nach einem fehlgeschlagenen Attentat gegen Präsident Gamal Abdel Nasser) verhaftet und schließlich, nach zehnjähriger Haft, Freilassung und der erneuten Festnahme, am 29. August 1966 hingerichtet. Qutbs Anhänger (unter ihnen sein Bruder Muhammad) flüchteten in den 1960er-Jahren nach Saudi-Arabien. Das saudische Königshaus empfing die Muslimbrüder mit offenen Armen, in der Hoffnung, dadurch den saudischen PanIslamismus, ein Gegenkonzept zum säkular-nationalistischen Pan-Arabismus, zu stärken. Die Muslimbrüder spielten eine entscheidende Rolle bei der 1962 gegründeten Muslimischen Weltliga, sowie der Weltversammlung der islamischen Jugend, die zehn Jahre später etabliert wurde. ${ }^{31}$ In Saudi-Arabien vermischte sich der Polit-Aktivismus der Muslimbruderschaft mit der saudischen Staatsdoktrin des Wahhabismus, einer puritanischen und extrem konservativen Auslegung des Islam, welche Feindschaft gegenüber allen Nichtmuslimen (sowie anderen muslimischen Strömungen wie Schiiten,

David von Drehle, A Lesson in Hate, in: Smithsonian Magazine, Februar 2006,

[https://www.smithsonianmag.com/history/a-lesson-in-hate-109822568/], eingesehen 4.11.2017.

${ }_{28}$ William E. Shepard, Sayyid Qutb's Doctrine of Jahiliyya, in: International Journal of Middle East Studies 35 (2003), Heft 4, S. 521-545; Sayed Khatab, „Hakimiyyah“ and ,Jahiliyya“ in the thought of Sayyid Qutb, in: Middle Eastern Studies 38 (2002), Heft 3, S. 145-170.

${ }_{29}$ Bassam Tibi, From Sayyid Qutb to Hamas. The Middle East Conflict and the Islamization of Antisemitism (The Yale Initiative for the Interdisciplinary Study of Antisemitism Working Paper Series 5), Yale 2010, S. 12-14.

${ }^{30}$ Camilla Adang u. a. (Hrsg.), Accusations of Unbelief in Islam. A Diachronic Perspective on Takfir, Leiden 2017.

31 Peter Mandaville, Muslim Networks and Movements in Western Europe, in: Pew Forum on Religion \& Public Life, September 2010, S. 27-32. 
Sufis oder Ahmadiyyas) propagiert. ${ }^{32}$ Während sich das saudische Königshaus das Netzwerk der Muslimbruderschaft zunutze machte, um die wahhabitische Ideologie weltweit in muslimischen Gemeinschaften (insbesondere auch westlichen Diasporas) zu fördern, betrachtete es den politischen Aktivismus der Muslimbrüder schon nach kurzer Zeit mit Argwohn. Angesichts der islamischen Revolution im Iran zu Beginn des Jahres 1979 und der Besetzung der Großen Moschee von Mekka durch Extremisten, die den Sturz des Königshauses forderten, zögerte Saudi-Arabien nach dem Einmarsch der Sowjetunion in Afghanistan deshalb nicht lange, sich als den primären Verteidiger des Islam darzustellen. Gemeinsam mit dem pakistanischen Geheimdienst ISI organisierte Saudi-Arabien finanzielle und logistische Unterstützung für arabische Freiwillige, die aus zahlreichen Ländern in den Dschihad gegen die ,gottlose Sowjetunion“ zogen. ${ }^{33}$ Zentrale Figuren in den saudischen Bemühungen waren Osama Bin Laden und Abdallah Azzam, die beiden Gründer von Al-Qaida. Mit tatkräftiger saudischer Unterstützung wurde der antisowjetische Dschihad zur Geburtsstunde der Globalen DschihadBewegung, die sich nach wenigen Jahren auch gegen das saudische Königshaus wandte. Ihr Ziel ist die Befreiung aller muslimischer Länder aus der Hand der Ungläubigen und der „Apostaten“-Regimes sowie die Etablierung eines globalen islamischen Kalifats. ${ }^{34}$ In den folgenden Abschnitten werden die Gemeinsamkeiten und Unterschiede sowie die komplexen Beziehungen zwischen Muslimbruderschaft und der Globalen DschihadBewegung weiter verdeutlicht.

\section{Hamas}

Die Wurzeln der Hamas reichen zurück bis in die Zeit des britischen Mandats für Palästina vor der Staatsgründung Israels. Im Jahr 1935 kam es zu einem Treffen von Abdel Rahman Al-Banna, dem Bruder Hassan Al-Bannas, mit dem für seine spätere Kollaboration mit dem Nationalsozialismus berüchtigten Jerusalemer Mufti Hajj Amin Al-Husseini. Dabei stimmte Hassan Al-Banna der Etablierung eines Zentralkomitees zur Unterstützung Palästinas zu, das gegen die britische Präsenz protestieren und die palästinensischen Nationalbestrebungen unterstützen sollte. ${ }^{35}$ Schließlich gründete Said Ramadan, der Vater von Tariq und Hani Ramadan und die zentrale Figur beim Aufbau des Muslimbruderschaft-Netzwerks im Westen, 1945 in Jerusalem eine Filiale der Muslimbruderschaft. ${ }^{36}$ Bereits zwei Jahre später verfügte die Muslimbruderschaft über

32 Carol E. B. Choksy/Jamsheed K. Choksy, The Saudi Connection. Wahhabism and Global Jihad, in: World Affairs Journal, Mai/Juni 2015, [http://www.worldaffairsjournal.org/article/saudi-connection-wahhabism-and-global-jihad], eingesehen 4.11.2017.

33 Thomas Hegghammer, Jihad in Saudi Arabia. Violence and Pan-Islamism since 1979, New York 2010, S. 26-30.

${ }^{34}$ Hegghammer, Jihad in Saudi Arabia.

35 Levitt, Hamas, S. 20.

36 Robert Dreyfuss, Cold War, Holy Warrior, in: Mother Jones, Januar/Februar 2006,

[http://www.motherjones.com/politics/2006/01/cold-war-holy-warrior/], eingesehen 5.11.2017; Ian Johnson, A 
25 Zweigstellen im Mandatsgebiet und zwischen 12.000 bis 20.000 Mitglieder. ${ }^{37}$ Während zwischen 1948 und 1967 die Muslimbruderschaft-Strukturen in Gaza in die ägyptische Mutterorganisation und jene im Westjordanland in den jordanischen Ableger integriert waren, führte der israelische Sieg im Sechstagekrieg und der damit einhergehende Gebietsgewinn zu einer Vereinheitlichung und zu einem deutlich gesteigerten Aktivismus der Muslimbrüder. ${ }^{38}$ Israel tolerierte diese Aktivitäten, da es sich ein Gegengewicht zum Terror der nominell säkularen Terrorgruppen, wie der Palästinensischen Befreiungsfront (PLO) und der Volkfront für die Befreiung Palästinas (PFLP), erhoffte. Anders als letztere waren die Muslimbrüder nicht an einer gewalttätigen Konfrontation interessiert, sondern auf die Islamisierung der palästinensischen Gesellschaft fokussiert. ${ }^{39}$ Der Ausbruch der Intifada im Dezember 1987 bestärkte allerdings eine radikale Fraktion der palästinensischen Muslimbrüder in der Überzeugung, dass der bewaffnete Kampf die einzige Lösung sei. Die Hamas war entstanden. In den folgenden Jahren entführte und tötete diese Organisation mehrere israelische Soldaten und wurde daraufhin von Israel verboten. ${ }^{40}$ Ironischerweise stärkte dieses Verbot die Glaubwürdigkeit der Hamas unter den Palästinensern, die der Organisation zuvor aufgrund der Tolerierung durch Israel misstraut hatten. Nachdem Ministerpräsident Yitzhak Rabin im Jahr 1992 über 400 Mitglieder der Hamas und des Palästinensischen Islamischen Dschihad (PIJ) in den Südlibanon deportieren ließ, nutzte die Hamas die Gelegenheit, um ihre militärischen Kapazitäten und Terrortaktiken in Ausbildungscamps der Hisbollah und einer PFLPFraktion (PFLP-Generalkommando von Ahmad Dschibril) weiterzuentwickeln. ${ }^{41}$ In den folgenden Jahren versuchte die Hamas gemeinsam mit dem PIJ und anderen radikalen PLO-Fraktionen, den Friedensprozess durch Terroranschläge zu torpedieren. Nicht zuletzt aufgrund ihrer kompromisslosen Haltung gegenüber Israel gewann sie Zuspruch unter den Palästinensern. Zugleich nutzte sie die 1990er-Jahre, um ihre Strukturen weiter auszubauen und ihren Einfluss, insbesondere in Gaza, zu konsolidieren. Der Ausbruch der Zweiten Intifada im Jahr 2000 war der Beginn einer beispielslosen Kampagne von Selbstmordanschlägen in Israel, von denen die meisten auf das Konto der Hamas gingen. ${ }^{42}$ Nach dem unilateralen Rückzug Israels aus dem Gaza im Jahr 2005, den viele Palästinenser der Hamas zuschrieben, beteiligte diese sich erstmalig an den palästinensischen Legislativwahlen - und gewann diese im Januar 2006 prompt. ${ }^{43}$ Doch nach anhaltenden Feindseligkeiten und Interessenskonflikten zwischen Hamas und der

Mosque in Munich. Nazis, the CIA, and the Rise of the Muslim Brotherhood in the West, San Diego 2011.

${ }^{37}$ Levitt, Hamas, S. 20.

38 Ebd., S. 21.

${ }^{39}$ Croituru, Hamas, S. 43-44.

40 Croituru, Hamas, S. 112-113.

${ }^{41}$ Croituru, Hamas, S. 127; Jonathan Schanzer, Hamas and Fatah. The Struggle for Palestine, New York 2008, S. 39.

42 Assaf Moghadam, Palestinian Suicide Terrorism in the Second Intifada. Motivations and Organizational Aspects, in: Studies in Conflict and Terrorism 26 (2003), Band 2, S. 65-92.

43 Peter Philipp, Zehn Jahre Hamas in Gaza, in: Deutschlandfunk, 25.1.2016, [http://www.deutschlandfunk.de/nahostkonflikt-zehn-jahre-hamas-in-palaestina.724.de.html?dram:article_id=343620], eingesehen 11.11.2017. 
Fatah unter der Führung von Mahmud Abbas, dem Präsidenten der Palästinensischen Autonomiebehörde (PA), übernahm die Hamas im Juni 2007 gewaltsam die Macht im Gazastreifen. ${ }^{44}$ Seither ist sie, trotz wiederholter innerpalästinensischer Versöhnungsversuche, die Alleinherrscherin in Gaza und bildet eine Parallelregierung zur PA im Westjordanland. ${ }^{45}$

\section{Antizionismus bei der Hamas}

Der bewaffnete Kampf bis zur Zerstörung Israels ist der raison d'être der Hamas. Bereits in der Einleitung ihres Gründungsdokuments lässt sie keinen Zweifel offen an ihrer Zielsetzung und zitiert Hassan Al-Banna: „Israel will exist and will continue to exist until Islam will obliterate it, just as it obliterated others before it. "46 Damit stellt die Hamas klar, dass es sich beim Kampf gegen Israel nicht einfach um einen territorialen Konflikt handelt, sondern um einen Krieg religiöser Natur, in der Tradition der Überlegungen von Rida, Al-Banna und Qutb. In Artikel 6 ihrer Gründungscharta heißt es: „Die Hamas strebt danach, das Banner des Islam über jedem Zentimeter Palästinas zu hissen “ und Artikel 11 beschreibt Palästina als „,islamisches Waqf“" (heiliges Land nur für Muslime) bis zum „Tag des Jüngsten Gerichts“. ${ }^{47}$ Die Hamas beschreibt den Kampf gegen Israel an mehreren Stellen als individuelle Pflicht (fard ayn, wie etwa das Beten und Fasten) eines jeden Moslems. Während der (defensive) Dschihad als individuelle Pflicht unter muslimischen Theologen schon immer umstritten war, popularisierte Abdullah Azzam, einer der Gründer von Al-Qaida, dieses Konzept in den 1980er-Jahren. ${ }^{48}$ Die HamasCharta ist zudem ein Musterbeispiel für die Verbindung von da’wa, also dem „Ruf zum Islam“, mit dem bewaffneten Kampf. Artikel 16 betont die Bedeutung einer islamischen Erziehung mit dem Studium von Koran, Sunna und islamischer Geschichte, aber auch „des Feindes, seiner Stärken und Schwächen, seinen Verbündeten, sowie seiner

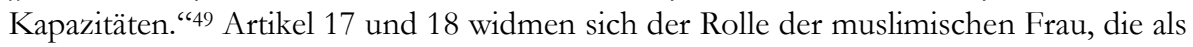
Schöpferin der Männer für die Erziehung der nächsten Generationen verantwortlich sei. ${ }^{50}$ In Artikel 19 wiederum ist die Rede von islamischer Kunst, die sich von der vorislamischen (jahili) Kunst unterscheide. Laut Hamas sind alle Formen von Kunst, egal

44 Ebd.

45 Jonathan Cook, Will Hamas-Fatah reconciliation deal succeed?, in: Al Jazeera, 13.10.2017,

[http://www.aljazeera.com/indepth/features/2017/10/hamas-fatah-reconciliation-deal-succeed-

171013064803703.html], eingesehen 11.11.2017.

${ }^{46}$ Hamas Covenant 1988. Yale Law School, The Avalon Project,

[http://avalon.law.yale.edu/20th_century/hamas.asp], eingesehen 11.11.2017.

47 Ebd.

48 Vgl. Majid Khadduri, War and Peace in the Law of Islam, Baltimore 1955, S. 60; Andrew McGregor, ,Jihad and the Rifle Alone." "Abdullah 'Azzam and the Islamist Revolution, in: The Journal of Conflict Studies 23 (2003), Band 2, [https://journals.lib.unb.ca/index.php/jcs/article/view/219/377], eingesehen 11.11.2017.

${ }^{49}$ Hamas Covenant 1988.

50 Ebd. 
ob Buch, Artikel, Predigt, Gedicht, Lied oder Theaterstück ein Mittel zur „,ideologischen Mobilisierung“". ${ }^{51}$ Der bewaffnete Kampf gegen Israel geht für die Hamas folglich einher mit einer umfassenden Islamisierung der palästinensischen Gesellschaft. Sie führt nicht nur regelmäßig Krieg gegen Israel, sondern erzieht Jugendliche in Gaza gemäß ihren Vorstellungen. ${ }^{52}$ In ihren Sommerlagern werden bereits Kinder auf den bewaffneten Kampf und den „glorreichen Märtyrertod“ vorbereitet. ${ }^{53}$ Daneben führt die Hamas ihren Kampf auch auf der politischen Ebene, sowohl in der palästinensischen Arena als auch international, wo sie unter anderem in der Europäischen Union kontinuierliche Lobbyarbeit betreibt. ${ }^{54}$ Seit Jahren behaupten Hamas-Unterstützer in Europa, die Organisation habe der Vernichtung Israels seit langem abgeschworen. Im Frühling 2017 präsentierte die Organisation eine neue Charta, die keine Referenzen mehr zu „Juden“ enthält und die Möglichkeit eines palästinensischen Staates in den Grenzen von 1967 andeutet. ${ }^{55}$ Nur wenige Tage später stellte Hamas-Mitbegründer Mahmoud Zahrar allerdings klar, dass die gewaltsame Befreiung „aller palästinensischen Gebiete“, worunter auch das israelische Kernland subsummiert wird, noch immer das Ziel der Organisation sei. ${ }^{56}$ Weitere Hamas-Anführer erklärten infolge, sie würden weder einer Entwaffnung, noch der Anerkennung Israels zustimmen. ${ }^{57}$

\section{Antisemitismus bei der Hamas}

Auch Antisemitismus spielt eine entscheidende Rolle in der Ideologie der Hamas. Ihre Judenfeindschaft speist sich dabei aus unterschiedlichen Quellen. Einerseits übernimmt die Hamas die Topoi einer jüdischen Weltverschwörung, die auch im Vernichtungs-

51 Ebd.

52 David Patrikarakos, Hamas Is Ready for War with Israel, in: Foreign Policy, 7.6.2016,

[http://foreignpolicy.com/2016/06/07/hamas-is-ready-for-war-with-israel-gaza-strip/], eingesehen 11.11.2017; Fares Akram/Jodi Rudoren, To Shape Young Palestinians, Hamas Creates Its Own Textbooks, in: The New York. Times, 3.11.2013, [http://www.nytimes.com/2013/11/04/world/middleeast/to-shape-young-palestinians-hamas-createsits-own-textbooks.html], eingesehen 11.11.2017.

53 Adnan Abu Amer, Thousands of teens enroll in Hamas summer camps, in: Al-Monitor, 18.8.2015, [http://www.almonitor.com/pulse/originals/2015/08/palestine-summer-camps-hamas-qassam-brigades-israel.html], eingesehen 11.11.2017.

54 The Global Muslim Brotherhood Watch Daily, European Hamas Support Group Announces Lobbying And Advocacy Course In Rotterdam, 21.1.2016, [https://www.globalmbwatch.com/2016/01/21/european-hamassupport-group-announces-lobbying-and-advocacy-course-in-rotterdam/], eingesehen 11.11.2017; CEPR und das europäische Netzwerk der Hamas, in: Audiatur-Online, 20.1.2014, [http://www.audiatur-online.ch/2014/01/20/ceprund-das-europaeische-netzwerk-der-hamas/], eingesehen 11.11.2017.

${ }^{55}$ Hamas in 2017. The document in full, in: Middle East Eye, 1.5.2017, [http://www.middleeasteye.net/news/hamascharter-1637794876], eingesehen 11.11.2017; Jack Khoury, Hamas Presents New Charter Supporting Palestinian State Along 1967 Borders, in: Haaretz, 1.5.2017, [https://www.haaretz.com/middle-east-news/palestinians/1.786754], eingesehen 11.11.2017.

56 Dov Lieber, Hamas assures critics Israel's destruction still its goal, in: The Times of Israel, 10.5.2017, [https://www.timesofisrael.com/hamas-assures-critics-israels-destruction-still-a-goal/], eingesehen 11.11.2017.

57 Jack Khoury, Hamas Leader in Gaza: We Will Neither Disarm nor Recognize Israel, in: Haaret\%, 19.10.2017, [https://www.haaretz.com/middle-east-news/palestinians/1.818235], eingesehen 11.11.2017. 
antisemitismus der Nazis zu finden waren. Andererseits wird die Judenfeindschaft aber religiös begründet, beispielsweise unter Berufung auf die berüchtigte Gharqad-Hadith, die in Artikel 7 ihrer Charta zitiert wird: „The Day of Judgement will not come about until Moslems fight the Jews (killing the Jews), when the Jew will hide behind stones and trees. The stones and trees will say O Moslems, O Abdulla, there is a Jew behind me, come and kill him. Only the Gharkad tree, (evidently a certain kind of tree) would not do that because it is one of the trees of the Jews. "58 Zudem heißt es in der Charta, dass „Israel, das Judentum und die Juden“ den Islam und die Muslime herausforderten (Art. 28) und dass ,islamische Gruppen“ (d. h. Gruppen wie die Hamas, im Gegensatz zu „säkularen“ Organisationen) am besten geeignet sind für den Kampf gegen die „kriegstreibenden Juden“ (Art. 32). ${ }^{59}$ Neben diesen religiösen Motiven finden sich in der Charta auch viele Elemente, die ihre Wurzeln im europäischen Antisemitismus des späten 19. und frühen 20. Jahrhunderts haben. Die „Zionisten“ hätten die Freimaurer, Rotarier und den Lions Club infiltriert (Art. 17) und würden hinter der französischen Revolution, dem Kommunismus sowie den beiden Weltkriegen stecken (Art. 22). ${ }^{60}$ Der offensichtliche Bezug auf die „Protokolle der Weisen von Zion“ - eine antisemitische Fälschung - wird in Artikel 32 explizit gemacht, der besagt, dass die Protokolle die „Pläne des Weltzionismus“ verkörpern würden. ${ }^{61}$ Der Antisemitismus der Hamas beschränkt sich nicht auf ihre Charta. Hamas-Mitbegründer Abdelaziz Al-Rantisi behauptete im Jahr 2003, der „falsche Holocaust“ sei die größte Lüge überhaupt, welche von den Zionisten erfunden wurde, um die Juden einzuschüchtern und so zur Emigration nach Palästina zu zwingen. ${ }^{62}$ Al-Rantisi argumentiert zudem, dass der Vergleich von Zionisten mit Nazis eine Beleidigung für letztere sei, da deren Verbrechen nur einen Bruchteil vom „Terror der Zionisten gegen das palästinensische Volk“ ausmachten. ${ }^{63}$ Ein weiteres klassisches Motiv in der antisemitischen Rhetorik der Hamas ist die Behauptung, Juden würden Christenblut für die Herstellung von Pessach-Matzot verwenden. Hamas-Sprecher Osama Hamdan stellte diese Behauptung im Juli 2014 auf und erklärte zudem, dies sei der Grund, weshalb die Israelis insbesondere Kinder ins Visier nehmen würden - es sei in ihrer zionistischen Psyche verankert. ${ }^{64}$ Ebenfalls im Juli 2014 bekräftigte ein HamasGeistlicher bei einer öffentlichen TV-Ansprache, dass man ,alle Juden vernichten“ werde. ${ }^{65}$

58 Hamas Covenant 1988.

59 Ebd.

60 Ebd.

61 Ebd.

62 Abdelaziz Al-Rantisi, The False Holocaust. The Greatest Lies Funded By The Zionists, in: Al-Risala, 21.11.2003, [https://www.memri.org/reports/hamas-leader-rantisi-false-holocaust-greatest-lies-funded-zionists], eingesehen 11.11.2017.

63 Ebd.

${ }^{64}$ Top Hamas Official: Jews Use Blood in their Passover Matzos, in: Israel National News, 31.7.2014, [http://www.israelnationalnews.com/News/News.aspx/183541], eingesehen 11.11.2017.

65 Palestinian Media Watch, Hamas cleric: We will “exterminate” the Jews "until the last one", in: YouTube, 30.07.2014, [https://youtu.be/UJNdDzweqbY], eingesehen 11.11.2017. 


\section{Al-Qaida}

Al-Qaida entstand Ende der 1980er-Jahre in Afghanistan unter der Führung von Osama Bin Laden und Abdullah Azzam. Bin Laden war der Sohn eines saudisch-jemenitischen Unternehmers, der über gute Kontakte zur saudischen Königsfamilie verfügte. ${ }^{66}$ Azzam wurde 1941 in der Nähe von Jenin geboren und zog nach dem Sechstagekrieg nach Jordanien, wo er sich 1969 der Muslimbruderschaft anschloss. ${ }^{67}$ Später erlangte er in Kairo ein Doktorat in islamischem Recht und begann, nach einem kurzen Aufenthalt in Saudi-Arabien in den frühen 1980er-Jahren, an der Islamischen Universität Islamabad in Pakistan zu unterrichten. ${ }^{68} 1984$ gab er diese Stelle auf, um sich auf den Jihad im benachbarten Afghanistan zu konzentrieren. Gemeinsam mit Bin Laden gründete Azzam in Peshawar das Makhtab Kadamat al-Mujaheddin, das Dienstleistungsbüro für Mujaheddin, welches arabische Jihadisten in Afghanistan finanziell und logistisch unterstützte. ${ }^{69}$ Nach Lawrence Wright gründeten Azzam, Bin Laden und weitere Anführer des Ägyptischen Islamischen Dschihads am 11. August 1988 Al-Qaida, wobei der Name der Organisation für mehrere Jahre geheim gehalten wurde. ${ }^{70} \mathrm{Zu}$ diesem Zeitpunkt war die Niederlage der Sowjetunion bereits absehbar und die arabischen Dschihadisten drängte es nach der „Befreiung“ aller muslimischer Länder. Allerdings waren sie sich nicht einig, wo der nächste Jihad zu führen sein würde. Während für Azzam die Befreiung Palästinas die höchste Priorität einnahm, betonte Ayman AlZawahiri, ein Anführer des Ägyptischen Islamischen Dschihads und der heutige Anführer Al-Qaidas, in Einklang mit der Lehre Sayyid Qutbs und der Takfir-Ideologie, die Notwendigkeit des Kampfes gegen „Apostaten-Regimes“, insbesondere demjenigen in seiner Heimat Ägypten. Sowohl Azzam als auch Zawahiri kämpften um die Gunst Bin Ladens, der Dank seinem Vater über beträchtliche finanzielle Mittel verfügte. ${ }^{71}$ Nachdem Azzam im November unter ungeklärten Umständen bei einem Bombenanschlag verstarb, fokussierte sich Al-Qaida anfänglich vor allem auf den von Zawahiri favorisierten „,nahen“ Feind und bekämpfte etwa die kommunistische Regierung im Jemen. ${ }^{72}$ Al-Qaida-Mitglieder und Sympathisanten beteiligten sich zudem an den Kriegen im Balkan. ${ }^{73}$ Spätestens gegen Mitte der 1990er-Jahre nahm Al-Qaida aber zunehmend den „fernen Feind“, also den Westen und insbesondere die USA, ins Visier. Al-Qaidas Anführer spekulierten, dass die arabischen „Apostaten-Regimes“ sich nur durch die

${ }^{66}$ Michael Scheuer, Osama Bin Laden, New York 2011, S. 21-22.

${ }^{67}$ Asaf Maliach, Abdullah Azzam, al-Qaeda, and Hamas. Concepts of Jihad and Istishhad, in: Military and Strategic Affairs 2 (2010), Band 2, S. 79.

${ }^{68}$ Ebd., S. 80.

${ }^{69}$ Ebd.

${ }^{70}$ Lawrence Wright, The Looming Tower. Al-Qaeda and the Road to 9/11, New York 2006, S. 131.

${ }^{71}$ Ebd. S. 8-9.

72 Byman, Al Qaeda, the Islamic State and the Global Jihadist Movement, S. 14.

73 Shaul Shay, Islamic Terror and the Balkans, New Brunswick 2009. 
Unterstützung der Vereinigten Staaten an der Macht halten konnten. ${ }^{74}$ Einen Rückzug der USA aus der Region würde demnach ihren Sturz zur Folge haben. Am 23. Februar 1998 veröffentlichten Bin Laden, Zawahiri sowie drei weitere Jihadisten aus Ägypten, Pakistan und Bangladesch einen Appell der „Weltislamischen Front“, der zum „Heiligen Krieg gegen Juden und Kreuzfahrer aufrief. ${ }^{75}$ In den Jahren darauf erfolgten die Anschläge gegen die US-Botschaften in Nairobi und Daressalam (1998), den Zerstörer USS Cole im Golf von Aden (2000), sowie Al-Qaidas größter Erfolg, die Anschläge vom 11. September 2001. Doch statt sich aus dem Nahen Osten zurückzuziehen, zerstörten die USA stattdessen die Infrastruktur von Al-Qaida in Afghanistan. In der Folge wandelte sich Al-Qaida von einer relativ hierarchisch strukturierten Organisation in eine weltweite Bewegung, mit Ablegern und Partnerorganisationen in zahlreichen Ländern. ${ }^{76}$ Seit Osama Bin Ladens Tod im Mai 2011 ist Ayman Al-Zawahiri Al-Qaidas neuer Anführer.

\section{Antizionismus bei Al-Qaida}

Der Jihad zur „Befreiung Palästinas“ war ein konstantes Thema für Bin Laden und Thomas Hegghammer hat bereits 2007 darauf hingewiesen, dass Palästina eine zentrale Rolle in der Strategie Al-Qaidas einnimmt. ${ }^{77}$ Operativ hingegen war Israel nie eine Priorität, doch dies lag nicht in seiner vermeintlich geringen Bedeutung begründet. Vielmehr sollten mit der Vertreibung der USA aus der Region und dem Sturz der arabischen Regimes überhaupt erst die Bedingungen geschaffen werden, um Palästina zu befreien. Von Bin Laden ist die Anekdote überliefert, dass er die Frage seines Sohnes Omar, warum Al-Qaida die USA statt Israel bekämpfe, wie folgt beantwortete: Israel und die USA seien wie ein Fahrrad mit einem hölzernen und einem eisernen Rad. Israel sei letzteres und deshalb sei es einfacher, zuerst das hölzerne Rad, d. h. die USA, zu zerstören. ${ }^{78}$ Tatsächlich halten sich dokumentierte Angriffe Al-Qaidas gegen israelische Ziele in Grenzen. Der vermutlich schwerste ereignete sich am 28. November 2002 während des Hanukkah-Fests in Kenia, als bei einem Selbstmordanschlag gegen ein Hotel mit israelischem Besitzer dreizehn Personen starben, darunter drei Israelis. ${ }^{79}$

\footnotetext{
74 Byman, Al Qaeda, the Islamic State and the Global Jihadist Movement, S. 16-17.

75 World Islamic Front, Jihad Against Jews and Crusaders, 23.2.1998, [https://fas.org/irp/world/para/docs/980223fatwa.htm], eingesehen 11.11.2017.

${ }^{76}$ Rick "Ozzie" Nelson/Thomas M. Sanderson, A Threat Transformed. Al-Qaeda and Associated Movements in 2011, 8.2.2011, [https://www.csis.org/analysis/threat-transformed], eingesehen 11.11.2017.

77 Maggie Michael, Bin Laden: Palestinian cause at heart of al-Qaeda's mission, in: USA Today, 16.5.2008, [http://usatoday30.usatoday.com/news/world/2008-05-16-bin-laden_N.htm], eingesehen 11.11.2017; Thomas Hegghammer, Osama bin Laden's true priorities, in: The Guardian, 3.12.2007,

[https://www.theguardian.com/commentisfree/2007/dec/03/osamabinladenstruepriorities], eingesehen 11.11.2017. 78 Scheuer, Bin Laden, S. 111-112.

${ }_{79}$ Marc Lacey/Benjamin Weiser, After Attack, Kenya Traces Qaeda's Trail in East Africa, in: The New York. Times, 1.12.2002, [http://www.nytimes.com/2002/12/01/world/after-attack-kenya-traces-qaeda-s-trail-in-east-africa.html], eingesehen 11.11.2017.
} 
Zeitgleich feuerten Terroristen eine Rakete auf ein Flugzeug der israelischen Fluggesellschaft Arkia ab, verfehlten aber ihr Ziel. Umstritten ist das Selbstmordattentat gegen die Mike's Place-Bar in Tel Aviv im Jahr 2003. Zwar bekannte sich die Hamas zu dem Anschlag, aber es gibt Hinweise darauf, dass die beiden britischen Selbstmordattentäter mit pakistanischem Hintergrund von Al-Qaida rekrutiert worden waren. ${ }^{80} 2005$ planten zudem Mitglieder der Salafistische Gruppe für Gebet und Kampf (GSPC), eine Partnerorganisation von Al-Qaida und der Vorgänger von Al-Qaida im Islamischen Maghreb (AQIM), ein El Al-Flugzeug beim Start in Genf abzuschießen, setzten ihr Vorhaben aber nie um. ${ }^{81}$

\section{Antisemitismus bei Al-Qaida}

Auch Antisemitismus spielt eine zentrale Rolle bei Al-Qaida. Im Appell der Weltislamischen Front vom Februar 1998 behauptet Al-Qaida, dass Juden die USA kontrollieren würden. ${ }^{82}$ Bin Laden wiederholte diese Behauptung in zahlreichen Interviews und verkündete zudem, die Juden würden auch die britische Regierung kontrollieren. ${ }^{83}$ Juden waren stets eines der wichtigsten gegnerischen Ziele für Al-Qaida und die Organisation rief wiederholt zum „Jihad gegen Juden“ auf. Inspire, das englischsprachige Magazin von Al-Qaida in der Arabischen Halbinsel, forderte im Mai 2012 dazu auf, Ziele anzugreifen, „wo sich Juden versammeln, ihre Anführer und wichtigsten Institutionen in Europa“". ${ }^{84}$ Zudem erklärte das Magazin, Juden seien neben „Polytheisten“ die ,größten Feinde“ des Islam. ${ }^{85}$ Brynjar Lia hat festgestellt, dass die Juden die einzige ,gegnerische“ Gruppe seien, die von Al-Qaida nie positiv erwähnt, bzw. denen nie ein Friedensangebot gemacht wurde. ${ }^{86}$ Al-Qaida führte zudem mehrere Anschläge gegen jüdische Ziele durch, etwa gegen die Synagoge von Djerba im September 2002 sowie gegen jüdische Gemeindezentren in Casablanca und Istanbul ein Jahr darauf. ${ }^{87}$ Auch der Anschlag von Mohammed Merah auf eine jüdische Schule in Toulouse im Jahr 2012 hat einen Bezug zu Al-Qaida. Obwohl Merah oftmals als sogenannter „Lone Wolf“ beschrieben wurde, trainierte er in Pakistan mit Jund Al-

80 Jonathan Schanzer, The Prospects of Al-Qaeda in Hamas-Controlled Gaza, in: Jewish Policy Center, 31.8.2007, [https://www.jewishpolicycenter.org/2007/08/31/the-prospects-of-al-qaeda-in-hamas-controlled-gaza/], eingesehen 11.11.2017.

81 Terrorists planned to blow up El Al plane, in: Ynetnews, 19.5.2006, [https://www.ynetnews.com/articles/0,7340,L3252772,00.html, eingesehen 11.11.2017.

82 World Islamic Front, Jihad Against Jews and Crusaders.

${ }^{83}$ Rahimullah Yusufzai, A Conversation with Terror, in: Time, 11.1.1999, [http://content.time.com/time/printout/0,8816,17676,00.html], eingesehen 11.11.2017.

84 Anti-Defamation League, Inspire Magazine Highlights "Jewish Enemy" To Recruit Jihadists, 31.5.2013, [https://www.adl.org/blog/inspire-magazine-highlights-jewish-enemy-to-recruit-jihadists], eingesehen 11.11.2017.

85 Ebd.

${ }^{86}$ Assaf Moghadam/Brian Fishman, Debates and Divisions within and around Al-Qa'ida, in: Moghadam/Fishman (Hrsg.), Self-Inflicted Wounds., S. 1-18, hier S. 9.

${ }^{87}$ Aaron Y. Zelin, Fifteen Years After the Djerba Synagoge Bombing, in: CTC Sentinel 10 (2017), Band 4. 
Khilafa, einer Al-Qaida-nahestehenden Gruppierung. Deren Anführer Moez Garsallaoui war anscheinend Merahs Mentor und ermutigte diesen, einen Anschlag in Frankreich durchzuführen. ${ }^{88}$

\section{Al-Qaida und Hamas}

Al-Qaida und Hamas verfügten zu ihren Anfangszeiten über ein Bindeglied in der Person von Abdallah Azzam, der bekannt war für seine Sympathien gegenüber der palästinensischen Terrororganisation und ihrem Gründer Scheich Ahmad Yassin. ${ }^{89}$ In einem Buch über die Hamas argumentierte er, nur diese sei in der Lage, Palästina zu befreien. ${ }^{90}$ Azzam unterstützte die Hamas auch finanziell und sammelte dafür Gelder während seiner USA-Reisen, wo er für die Unterstützung des antisowjetischen Jihads warb. ${ }^{91}$ Im Gegenzug führte die Hamas im Dezember 1989 einen Generalstreik durch, aus Protest gegen die Ermordung Azzams. ${ }^{92}$ Zudem benannte sie verschiedene Einrichtungen und Einheiten nach ihm, etwa ihren militärischen Flügel im Westjordanland (später vereinheitlicht in Al-Qassam-Brigaden) sowie die Shahid Dr. Abdullah Azzam-Militärakademie, die 2006 in Gaza eröffnet wurde. ${ }^{93}$ Allerdings hat sich in den vergangenen fünfzehn Jahren das Verhältnis zwischen der Hamas und Al-Qaida sehr verschlechtert. Al-Qaida kritisierte erstere für deren Beteiligung an den palästinensischen Legislativwahlen im Jahr 2006 sowie ihren positiven Bezug auf den palästinensischen Nationalismus. ${ }^{94}$ An dieser Stelle wird der Unterschied zwischen der Hamas als Vertreterin der Muslimbruderschaft und der salafistisch-jihadistischen AlQaida deutlich. Al-Qaida warf der Hamas darüber hinaus vor, das palästinensische Volk zu verraten, da sie bereit sei, Waffenstillstände mit Israel einzugehen. ${ }^{95}$ Zudem kritisierte Al-Qaida die Hamas für ihren mangelnden Antiamerikanismus, die höchstens partielle Einführung der Scharia in Gaza sowie ihre Zusammenarbeit mit dem Iran und der Hisbollah. ${ }^{96}$ Umgekehrt wirft die Hamas Al-Qaida vor, dass ihre Unterstützung für die Palästinenser außer wohlfeilen Erklärungen wenig Konkretes zu bieten habe. ${ }^{97}$ Die Hamas war stets darauf bedacht, den Einfluss von Al-Qaida und den ihr nahestehenden

88 Delphine Byrka, On a retrouvé le mentor taliban de Mohamed Merah, in: Paris Match, 12.9.2012,

[http://www.parismatch.com/Actu/Societe/On-a-retrouve-le-mentor-taliban-de-Mohamed-MeRah-161129], eingesehen 11.11.2017.

${ }^{89}$ Maliach, Abdullah Azzam, al-Qaeda, and Hamas, S. 87-88.

${ }^{90}$ Ebd., S. 88.

${ }^{91}$ Ebd.; Thomas Hegghammer, 'Abdallāh 'Azzām and Palestine, in: Welt des Islams 53 (2013), Band 3/4, S. $377-378$.

92 Maliach, Abdullah Azzam, al-Qaeda, and Hamas, S. 88.

93 Ebd., S. 89.

$94 \mathrm{Paz}$, Jihadis and Hamas, S. 188-189.

95 Ebd., S. 192-193.

${ }^{96}$ Ebd., S. 189-192.

97 Ebd., S. 200. 
Gruppierungen im Gazastreifen einzuschränken. Wiederholt ging sie massiv gegen salafistisch-dschihadistische Organisationen vor, oftmals mit tödlichem Ausgang. ${ }^{98}$

\section{Islamischer Staat}

Spätestens seit der Islamische Staat (IS) bei seiner Offensive im Juni 2014 Mossul, eine der größten Städte des Iraks, einnahm, ist er zum berüchtigtsten und medial am meist beachteten Vertreter der globalen Dschihad-Bewegung avanciert. Seine Geschichte geht aber viel weiter zurück. Im Jahr 1999 etablierte der jordanische Dschihadist Abu Musab Al-Zarqawi, nachdem er im Rahmen einer Generalamnestie freigekommen war, mit der finanziellen Unterstützung Osama bin Ladens ein Trainingscamp im afghanischen Herat. ${ }^{99}$ Nach der US-Invasion in Afghanistan floh Zarqawi via Iran in den Irak, von wo er sein Netzwerk namens Jama'at Tawhid w'al Jihad (Organisation für Monotheismus und Dschihad) ausbaute. Dazu gehörte unter anderem auch die sogenannte TawhidGruppe, welche in Deutschland Anschläge auf jüdische und israelische Ziele plante, diese Pläne aber nie umsetzte. ${ }^{100}$ Nach dem US-Einmarsch im Irak waren Zarqawi und sein Netzwerk nach kurzer Zeit einer der zentralen Akteure unter den zahlreichen Dschihadistengruppen. ${ }^{101}$ Obwohl Zarqawi auch im Irak auf finanzielle Hilfe durch AlQaida zählte, bestand er auf seiner Unabhängigkeit und weigerte sich, seine Gruppe AlQaida offiziell anzuschließen. ${ }^{102}$ Erst im Oktober 2004 schwor er den Treueeid auf Bin Laden und benannte seine Gruppe offiziell in Al-Qaida in Irak (AQI) um. ${ }^{103}$ Allerdings führte Zarqawis Fokus auf das Entfachen eines konfessionellen Kriegs mit den irakischen Schiiten zu Konflikten mit der Al-Qaida-Führung, die Angriffe auf die USund Koalitionstruppen bevorzugte. Zawahiri kritisierte Zarqawis Brutalität, die sich auch gegen sunnitische Irakis richtete, in einer privaten Korrespondenz. ${ }^{104}$ Zarqawi zeigte sich davon wenig beeindruckt und nachdem er bei einem US-Angriff 2006 ums Leben kam, hielten seine Nachfolger an seiner Strategie fest und benannten die Organisation in Islamischer Staat im Irak (ISI) um. ${ }^{105}$ Die willkürliche Gewalt führte letztlich zu einer Gegenreaktion unter vielen sunnitischen Stämmen, dem sogenannten Anbar Erwachen, welches gemeinsam mit der US-amerikanischen Truppenaufstockung 2007, den ISI in

98 Peter Beaumount, Hamas destroys al-Qaida group in violent Gaza battle, in: The Guardian, 16.8.2009, [https://www.theguardian.com/world/2009/aug/15/hamas-battle-gaza-islamists-al-qaida], eingesehen 11.11.2017.

${ }_{99}$ Brian Fishman, The Master Plan. ISIS, Al-Qaeda, and the Jihadi Strategy for Final Victory, New Haven 2016, S. 8 und 19-21.

100 Fishman, The Master Plan, S. 27-28.

${ }^{101}$ Byman, Al Qaeda, the Islamic State and the Global Jihadist Movement, S. 166.

102 Ebd.

103 Ebd.

104 Zawahiri's Letter to Zarqawi (English Translation), in: Combating Terrorism Center, o. D., [https://ctc.usma.edu/wpcontent/uploads/2013/10/Zawahiris-Letter-to-Zarqawi-Translation.pdf], eingesehen 11.11.2017.

105 Byman, Al Qaeda, the Islamic State and the Global Jihadist Movement, S. 166. 
die Defensive drängte. ${ }^{106}$ Der Abzug der US-Truppen 2011 und die darauffolgende Repression Bagdads gegen die sunnitische Bevölkerung des Irak sowie der Ausbruch des syrischen Bürgerkriegs eröffneten dem ISI neue Möglichkeiten. Sein neuer Anführer, Abu Bakr Al-Baghdadi, entsandte einige ISI-Kämpfer unter der Führung von Abu Mohammed Al-Jolani nach Syrien, um dort eine Präsenz zu etablieren. ${ }^{107}$ Jabhat AlNusra, so der Name des syrischen Ablegers, avancierte in Kürze zu einer der effektivsten Rebellengruppen. Im April 2013 erklärte Baghdadi, Jabhat Al-Nusra sei ein Teil des ISI und verkündete die Vereinigung beider Gruppen zum Islamischen Staat im Irak und in Syrien (ISIS). ${ }^{108}$ Das lehnten sowohl die Jabhat Al-Nusra, welche sich als offizieller AlQaida-Ableger in Syrien verstand, als auch die Kern-Al-Qaida unter der Führung von Zawahiri ab. Dies entfachte einen brutalen Konflikt zwischen ISIS und Jabhat Al-Nusra, in welchem ersterer zumindest einstweilen die Oberhand gewann. ${ }^{109}$ Die ISIS-Offensive im Jahr 2014 brachte der Organisation massive Gebietsgewinne sowohl im Irak als auch in Syrien. Baghdadi änderte den Namen der Organisation zu Islamischer Staat (IS) und erklärte sich selbst zu dessen Kalifen und dem Anführer aller Muslime. Seit 2014 hat der IS zahlreiche Ableger in verschiedenen Ländern etabliert, darunter viele ehemalige Partnerorganisationen von Al-Qaida, welche die Seite wechselten. Als er in seinem Kerngebiet militärisch immer stärker unter Druck geriet, entfesselte der IS weltweit eine beispielslose Terrorwelle, die auch nach - mittlerweile massiven - Gebietsverlusten anhält. ${ }^{110} \mathrm{Ob}$ und wie sich der IS von seiner militärischen Niederlage im Irak und in Syrien erholen wird, bleibt abzuwarten.

\section{Antizionismus beim Islamischen Staat}

Ähnlich wie bei Bin Laden spielte der israelisch-palästinensische Konflikt eine wichtige Rolle in der Rhetorik Zarqawis, demzufolge der „Islamische Staat im Irak das Tor zur Befreiung des gestohlenen Palästinas" sei. ${ }^{111}$ Die zweite Ausgabe von Dabiq, einem englischsprachigen Online-Magazin des IS, verkündete ,it is only a matter of time and patience before it reaches Palestine to fight the barbaric jews and kill those of them hiding behind the gharqad trees - the trees of the jews". ${ }^{112}$ Allerdings beschränken sich die antiisraelischen Aktivitäten des IS ausschließlich auf Rhetorik. Im Vergleich zu Al-Qaida nimmt Israel beim IS eine geringere Priorität ein. In einer Audiobotschaft Abu Bakr AlBaghdadis vom November 2014, in der dieser unter anderem die wichtigsten Feinde des

\footnotetext{
106 Austin Long, The Anbar Awakening, in: Survival 50 (2008), Band 4, S.67-94.

107 Byman, Al Qaeda, the Islamic State and the Global Jihadist Movement, S. 166-167.

108 Ebd, S. 168.

109 Ebd., S.168-169.

110 Andrew Watkins, Losing Territory and Lashing Out. The Islamic State and International Terror, in: CTC Sentinel 9 (2016), Band 6, S. 14-18.

111 Paz, Jihadis and Hamas, S. 196.

112 Foreword, in: Dabiq (2014), Heft 2, S. 4.
} 
IS aufzählte, wurde Israel nicht einmal erwähnt. ${ }^{113}$ Stattdessen standen an erster Stelle die Schiiten, gefolgt von den arabischen „Apostaten-Regimes“ und schließlich den „Kreuzfahrern“, also dem Westen. Erst im Dezember 2015 drohte Baghdadi in einer Audiobotschaft, er habe die Juden nicht vergessen und Palästina werde ihr Friedhof sein. ${ }^{114}$ Zwei Monate zuvor hatte die Organisation erstmals ein Video auf Hebräisch veröffentlicht, in dem ein IS-Kämpfer ankündigte, der IS würde die Al-Aqsa Moschee als Eroberer betreten. ${ }^{115}$ In einem Artikel im arabischsprachigen Al-Naba Magazin stellte der IS im März 2016 allerdings klar, dass der Jihad in Palästina keine wichtigere Rolle einnehmen würde als der Jihad andernorts. ${ }^{116}$ Diese geringe Priorität zeigt sich ebenfalls in der Abwesenheit von IS-Angriffen gegen Israel. Eine der wenigen Ausnahmen ist ein Gefecht an der Grenze zu Syrien im November 2016, in dessen Verlauf israelische Soldaten vier Kämpfer der Khalid Ibn Al-Walid-Armee, einer dem IS-nahestehenden Organisation, töteten. ${ }^{117}$ Abgesehen davon ist davon auszugehen, dass einige ISAnhänger in Gaza seit dem Sommer 2014 in sporadische Raketenangriffe gegen Israel involviert waren. Auch diese dürften aber ohne Absprache mit der IS-Führung erfolgt sein. Eine höhere Gefahr geht möglicherweise vom IS-Ableger auf der Sinai-Halbinsel aus. Israelische Sicherheitskräfte gingen im September 2016 davon aus, dass dieser in der nahen Zukunft einen Angriff gegen Israel durchführen würde. ${ }^{118}$

\section{Antisemitismus beim Islamischen Staat}

Analog zu Hamas und Al-Qaida ist die IS-Rhetorik geprägt von antisemitischen Elementen. Dessen mittlerweile getöteter Sprecher Abu Muhammad al-Adnani bezeichnete in einer Audiobotschaft von September 2014 Präsident Obama als ein „Maultier der Juden“ und behauptete, die Juden hätten gemeinsam mit den Kreuzfahrern hunderttausende Muslime getötet. ${ }^{119}$ Zudem zitierte er eine Stelle im Koran, gemäß der

113 Even If The Disbelievers Despise Such - Baghdadi's latest speech (Transcript), in: Terrorism Research and Analysis Consortium, o. D., [https://www.trackingterrorism.org/chatter/even-if-disbelievers-despise-such-baghdadis-latestspeech-transcript], eingesehen 11.11.2017.

114 Denver Nicks, ISIS Leader Reportedly Warns Israel. „We Are Getting Closer to You“, in: TIME, 26.12.2015, [http://time.com/4161623/isis-leader-baghdadi-message-israel/], eingesehen 11.11.2017.

115 Daniel J. Roth, Watch: Hebrew-Speaking ISIS-Jihadi Threatens Israel, Jews Worldwide in New Video, in: The Jerusalem Post, 23.10.2015, [http://www.jpost.com/Arab-Israeli-Conflict/Hebrew-speaking-ISIS-jihadi-threatensIsrael-Jews-worldwide-in-new-video-429884], eingesehen 11.11.2017.

116 ISIS: Jihad In Palestine Does Not Take Precedence Over Jihad Elsewhere, in: MEMRI, 22.3.2016, [https://www.memri.org/reports/isis-jihad-palestine-does-not-take-precedence-over-jihad-elsewhere\#_edn3], eingesehen 11.11.2017.

117 Judah Ari Gross, First IDF-Islamic State clash sends Israeli message: Don't mess with us, in: The Times of Israel, 27.11.2016, [http://www.timesofisrael.com/first-idf-islamic-state-clash-unlikely-a-gamechanger-experts-say/], eingesehen 11.11.2017.

118 Israel 'bracing for attack' by IS in Sinai in next 6 months, in: The Times of Israel, 10.9.2016, [http://www.timesofisrael.com/israel-bracing-for-attack-by-is-in-sinai-in-next-6-months/], eingesehen 11.11.2017.

119 Yara Bayoumy, Islamic State urges attacks on U.S., French citizens, taunts Obama, in: Reuters, 22.9.2014, [http://www.reuters.com/article/us-iraq-crisis-adnani/islamic-state-urges-attacks-on-u-s-french-citizens-taunts- 
die Juden versuchen würden, die Muslime von ihrem Glauben abzubringen. Auch das bereits erwähnte IS-Video in hebräischer Sprache ist geprägt von Antisemitismus. Der hebräischsprachige IS-Kämpfer kündigt darin an, dass der Islamische Staat die ganze Welt von der „Krankheit“ der Juden reinigen werde. ${ }^{120}$ Wenig verwunderlich finden sich auch in den IS-Publikation wie Dabiq antisemitische Topoi wie die Gharqad-Hadith und zahlreiche Verschwörungstheorien, wonach Juden die US-Regierung kontrollieren würden. ${ }^{121}$ Allerdings nehmen die Juden im Vergleich mit der IS-Propaganda gegen Schiiten, Apostaten und Christen eine fast schon untergeordnete Rolle ein. Dies hat ISAnhänger in Europa allerdings nicht davon abgehalten, gezielte Terrorakte gegen jüdische Ziele zu begehen, etwa gegen das jüdische Museum in Brüssel 2014, den Hyper Cacher-Supermarkt in Paris im Januar 2015 sowie gegen eine Synagoge in Kopenhagen im Februar 2015. ${ }^{122}$ Zudem habe laut anonymen Quellen mindestens einer der Attentäter des Anschlags am Brüsseler Flughafen im März 2016 gezielt orthodoxe Juden ins Visier genommen. ${ }^{123}$

\section{Der Islamische Staat und die Hamas}

Das Verhältnis zwischen dem IS und der Hamas ist mindestens so kompliziert wie jenes zwischen letzterer und Al-Qaida. Der IS kritisierte die Hamas wiederholt für ihren Nationalismus und ihre unzureichende Umsetzung der Scharia in Gaza. Verglichen mit jener Al-Qaidas an der Hamas fällt die Kritik aber noch schärfer aus und in verschiedenen Publikationen wurden Hamas und Muslimbruderschaft als „Apostaten“ und „Verräter gegenüber dem Islam“ bezeichnet. ${ }^{124}$ Während dem Gaza-Krieg von 2014 verbrannten ISIS-Kämpfer in Syrien Palästinenserflaggen, ein klarer Affront gegenüber der Hamas. ${ }^{125}$ Im Gegenzug verurteilte die Hamas den Anschlag auf die Charlie Hebdo-Redaktion im Januar 2015, wobei sie die jüdischen Opfer des Hyper Cacher Anschlags unerwähnt ließ, sowie die multiplen Terrorakte in Paris im November 2015. ${ }^{126}$ Der Hamas-nahe

obama-idUSKCNOHH1MB20140922], eingesehen 11.11.2017.

120 Roth, Hebrew-Speaking ISIS-Jihadi Threatens Israel.

121 Anti-Defamation League, Anti-Semitism: A Pillar of Islamic Extremist Ideology, 2015, S. 3-4,

[https://www.adl.org/sites/default/files/documents/assets/pdf/combating-hate/Anti-Semitism-A-Pillar-of-

Islamic-Extremist-Ideology.pdf], eingesehen 11.11.2017.

122 Ebd., S. 4.

123 Lachlan Carmichael, Brussels airport bombers targeted Jews, Americans, investigators say, in: The Times of Israel, 24.1.2017, [http://www.timesofisrael.com/brussels-airport-bombers-targeted-jews-americans-investigators-say/], eingesehen 11.11.2017.

124 Cole Bunzel, From Paper State to Caliphate: The Ideology of the Islamic State (The Brookings Project on U.S. Relations with the Islamic World Analysis Paper 19), Washington D.C. 2015, S. 10.

125 Ali Mamouri, Why Islamic State has no sympathy for Hamas, in: Al-Monitor, 29.7.2014, [https://www.almonitor.com/pulse/originals/2014/07/islamic-state-fighting-hamas-priority-before-israel.html],

eingesehen 11.11.2017.

126 Nidal al-Mughrabi, Hamas condemns France attacks, says no justification for ,killing innocents', in: Reuters, 11.1.2015, [http://www.reuters.com/article/us-france-shooting-hamas/hamas-condemns-france-attacks-says-nojustification-for-killing-innocents-idUSKBNOKJOMP20150111], eingesehen 11.11.2017; Hamas, Islamic Jihad 
Muslimbruderschaft-Geistliche Scheich Yussuf Al-Qaradawi erklärte bereits 2014 das ISKalifat für illegitim. ${ }^{127}$ Zudem geht die Hamas, wie schon früher gegen Al-QaidaSympathisanten, entschlossen gegen IS-Anhänger vor. Im Mai 2015 zerstörte sie eine Salafistenmoschee in Gaza und verhaftete wiederholt IS-Sympathisanten. IS-Anhänger in Gaza erklärten infolge, die Hamas sei schlimmer als Israel. ${ }^{128}$ Auf der anderen Seite arbeitet die Hamas eng mit dem IS-Ableger auf der Sinai Halbinsel zusammen. Dessen Kämpfer erhielten etwa medizinische Versorgung in Gaza sowie militärisches Training durch die Qassam-Brigaden. ${ }^{129}$ Die Hamas erlaubte dem IS-Ableger zudem die Eröffnung eines Propaganda-Büros in Gaza, beliefert dessen Kämpfer mit Waffen und besteuert IS-Schmuggeltunnels zwischen Gaza und Ägypten. ${ }^{130}$

\section{Schlussfolgerung}

Wie dieser Beitrag aufgezeigt hat, spielen Antisemitismus und Antizionismus sowohl bei Hamas als auch bei Al-Qaida und IS eine wichtige Rolle in Ideologie und Praxis. Zugleich lassen sich bei den drei Organisation erhebliche Unterschiede ausmachen, insbesondere bei konkreten antisemitischen oder antizionistischen Aktivitäten (die Rhetorik dagegen ist bei allen mehr oder weniger dieselbe). Für die Hamas bleibt die Zerstörung Israels ihr ultimatives Ziel und folglich fokussiert das Gros ihrer Anstrengungen, d. h. sowohl ihre politischen als auch militärischen Kapazitäten, darauf. Zugleich ist sie bemüht, trotz fundamental antisemitischer Rhetorik, ihre Angriffe seit Ende der zweiten Intifada auf ihrer Ansicht nach - „legitime“ Ziele, d. h. im Allgemeinen „Zionisten“ und dabei insbesondere Soldaten und Siedler, zu beschränken. So ist bei der Hamas, anders als bei den säkularen palästinensischen Terrorgruppen, kein Anschlag außerhalb des israelischen bzw. palästinensischen Gebiets bekannt. Während das Konzept des Juden als des totalen Feindbilds im kosmischen Kampf zwischen Gut und Böse zwar durchaus eine Rolle spielt, zeichnen sich die Aktivitäten der Hamas durch einen gewissen Pragmatismus aus, der ihr in den Augen der Weltöffentlichkeit Legitimation für ihre Sache verleihen soll.

condemn Paris attacks, in: The Times of Israel, 14.11.2015, [http://www.timesofisrael.com/hamas-islamic-jihadcondemn-paris-attacks/], eingesehen 11.11.2017.

127 Shafik Madhai, Muslim leaders reject Baghdadi's caliphate, in: Al Jazeera, 7.7.2014,

[http://www.aljazeera.com/news/middleeast/2014/07/muslim-leaders-reject-baghdadi-caliphate-

20147744058773906.html], eingesehen 11.11.2017.

128 Joanna Paraszczuk, As Tensions Rise, Pro-IS Group In Gaza Complains Hamas ,Worse Than Israel', in: Radio Free Europe/Radio Liberty, 5.5.2015, [https://www.rferl.org/a/islamic-state-group-in-gaza-says-hamas-worse-thanisrael/26995991.html], eingesehen 11.11.2017.

129 Michel Wyss, Hamas und der Islamische Staat. Zwischen Kooperation und Repression, in: Audiatur-Online, 27.1.2016, [http://www.audiatur-online.ch/2016/01/27/hamas-und-der-islamische-staat-zwischen-kooperationund-repression/], eingesehen 11.11.2017.

130 Avi Issacharoff, Hamas, Islamic State resume close cooperation despite pressure from Cairo, in: The Times of Israel, 15.1.2017, [http://www.timesofisrael.com/hamas-islamic-state-resume-close-cooperation-despite-pressure-fromcairo/], eingesehen 11.11.2017. 
Al-Qaida und IS dagegen kümmern sich nicht um solche - für die Hamas politisch relevanten - Distinktionen. Stattdessen haben beide gemäß ihrer antisemitischen Rhetorik bislang vorwiegend jüdische Ziele außerhalb Israels ins Visier genommen (selbstverständlich liegt auch dies teilweise einem operationellen Pragmatismus zugrunde, da solche Ziele einfacher anzugreifen sind). Interessant ist dabei, dass für den IS sowohl die Juden als auch Israel als Feindbilder eine geringere Rolle spielen als bei Al-Qaida, bei der diese zentral waren. Dies dürfte einerseits mit einem Bedürfnis nach Abgrenzung gegenüber Al-Qaida zusammenhängen, andererseits aber auch mit der operationellen Ausrichtungen der beiden Organisation: der „ferne Feind“ bei Al-Qaida, bzw. der „nahe Feind" beim IS. Schließlich lassen sich anhand dieses Vergleichs die Unterschiede und Gemeinsamkeiten zwischen der Muslimbruderschaft, vertreten von der Hamas, und der globalen Dschihad-Bewegung, repräsentiert durch Al-Qaida und IS, nachvollziehen. Die Hamas fokussiert (einstweilen) auf ein örtlich beschränktes Ziel und verbindet zu dessen Erreichung Nationalismus und Islam. Neben dem Einsatz von Gewalt gibt sie sich politisch pragmatisch und partizipiert gar an demokratischen Prozessen. Für Al-Qaida und den IS hingegen gibt es, in den Worten Abdallah Azzams, ,nur das Gewehr und den Dschihad“. Jegliches politische Engagement außerhalb des bewaffneten Kampfs gilt für sie als unislamisch und der salafistischen Doktrin widersprechend. Folglich sind für AlQaida und IS die Hamas und überhaupt die Muslimbruderschaft Verräter an ihrer Religion (was aber, wie aufgezeigt, taktische Kooperationen zwischen beiden Seiten nicht verunmöglicht). Einig sind sich alle drei einzig und allein in ihrer Feindschaft gegen Israel und das Judentum. 
